\title{
Image Reconstruction Using WTA-ICA Model in Contourlet Transform Domain
}

\author{
Li Shang \\ College of Electronic Information Engineering \\ Suzhou Vocational University \\ Suzhou, China \\ e-mail: \{s10930\}@ jssvc.edu.cn
}

\author{
Zhanli Sun \\ College of Electrical Engineering and Automation \\ Anhui University \\ Hefei, China \\ e-mail: zhlsun@yahoo.com.cn
}

\begin{abstract}
A new image reconstruction method using the WTAICA model in contourlet transform domain is discussed in this paper. WTA-ICA is in fact an sparse ICA algorithm, and is simpler and faster under high dimensional computational requirements. Contourlet transform can offer a flexible multiresolution and directional decomposition for images and embody well image structure. Here, for a given image, the contourlet transform is first used to obtain low and high frequency sub-band images at two layers, where each layer behaves four directions. Then, WTA-ICA model is used in contourlet domain to extract low frequency sub-band image features and each layer's high frequency sub-band image features. Further, considered the fusion technique between each layer's high frequency sub-band features, as well as that between the fused high frequency sub-band image features and the first level's low frequency features, the total image fused features can be obtained. Using this fusion feature, an image can be reconstructed well. The quality of reconstructed images is measured by SNR criterion, and compared with WTA-ICA model, the experimental results shown that our method is indeed efficient in image reconstruction task.
\end{abstract}

Keywords- WTA-ICA;Sparseness, $l^{\infty}$ norm Contourlet transform; Feature fusion; Image reconstruction

\section{INTRODUCTION}

The goal of image reconstruction intends to recover the original ideal image from its given bad version, such as one contaminated by noise, or blurred by atmospheric turbulence [1]. In the process of image reconstruction, both denoising and maintaining of the detail about the image is required. However, in most cases, the both are mutually conflicting in application. To this day, many methods of image reconstruction has been developed to solve this confliction [2-4], such as filter based methods [2] wavelet based methods [3], sparse representation based methods and so on [4]. In these methods, sparse representation based methods has been used widely at present. This method can reduce hardly computation complexity. While winner-take-all (WTA) based independent component analysis (ICA) model (denoted by ICA model here) is in fact a sparse representation model. It utilizes the $l^{\infty}$ norm as the independence and sparse measure criterion, and compared with basic ICA model, it is much simpler and faster under high dimensional computational requirements. To ensure the sparseness and the high frequency details of an image, the contourlet transform is first used to preprocess it, and then, in the contourlet domain, WTA-ICA model is used to extract all low frequency and high frequency sub-band images' features. For high frequency sub-band features at each layer, the weighted average feature fusion technique is used, and then the high frequency fusion features are obtained. Further, the fusion method between this high frequency fusion features and the first level's low frequency features are again discussed, and the fusion features obtained are used as the total image features. Finally, utilizing the total image features, an image can be reconstructed successfully [4]. This method can well both retain the low and high frequency information of images. To testify the effect of our method, the image reconstruction task using WTA-ICA model are also discussed in this paper. Experimental results testify that the method explored by us is indeed efficient.

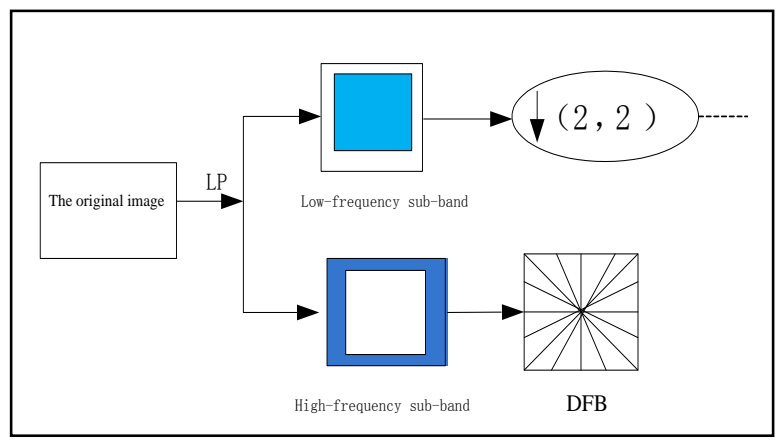

Figure 1. The illustration of contourlet transform process.

\section{CONTOURLET TRANSFORM}

Contourlet transform method was proposed by M. N. Do et al [6-7] on the basis of wavelet transform method. This transform can offer a directional multi-resolution image representation, which can efficiently capture and represent smooth object boundaries in natural images, since it allows for a different number of directions at each scale [5]. The contourlet transform is constructed as a combination of the Laplacian Pyramid [5] and the 2-D directional filter banks (DFB) that can be maximally decimated while achieving perfect reconstruction [6]. And this transform process can be simply generalized as two steps [7]: (1) Firstly, to catch all singular points in an image, the multi-scale decomposition is realized by using the LP filter. (2) For the HP sub-band images, the DFB are applied to them in order to make all 
singular points in the same direction composed into a contourlet. The DFB are designed to capture the high frequency components of images, and are efficiently implemented via an $l$-level tree-structured decomposition, which can lead to $2^{l}$ sub-bands with wedge-shaped frequency partitioning as shown in Fig.1, and each sub-band represents a direction in Fig.1.

\section{WTA-ICA MODEL}

\section{A. The Cost Function with $l^{\infty}$ norm based Sparseness}

Assumed that a random vector $y$ has zero mean value and unit variance, so its sparseness to $l^{p}$ norm criterion is defined as follows [8]

$$
\operatorname{Sparse}(y)=-E\left\{\left(\sum_{i}\left|y_{i}^{p}\right|\right)^{\frac{1}{p}}\right\}
$$

In Equation (1), when $p \rightarrow \infty$, the limit of $\left(\sum_{i}\left|y_{i}^{p}\right|\right)^{1 / p}$ is written as follows [8]:

$$
\lim _{p \rightarrow \infty}\left(\sum_{i}\left|y_{i}^{p}\right|\right)^{1 / p}=\max _{i}\left\{\left|y_{i}\right|\right\}
$$

Thus, for the $l^{\infty}$ norm sparseness measure function, the optimization process is relatively easy, which is defined the following form

$$
J(y)=E\left\{\lim _{p \rightarrow \infty}\left(\sum_{i}\left|y_{i}^{p}\right|\right)^{1 / p}\right\}=E\left\{\max _{i}\left\{\left|y_{i}\right|\right\}\right\}
$$

and for mathematical convenience, let $\left|y_{j}\right|$ be replaced with $\left|y_{j}\right|^{2}=\left(w_{j}^{T} x\right)^{2}$ (here $x$ is also a random vector and $w_{j}$ is the column vector of weighted matrix $W$ ), thus, Equation (3) can be rewritten as [8]:

$$
J(W)=E\left\{\max \left[\left(w_{j}^{T} x\right)^{2}\right]\right\}=\int \max _{j}\left[\left(w_{j}^{T} x\right)^{2}\right] p(x) d x
$$

Let $c=\arg \max _{j}\left[\left(w_{j}^{T} x\right)^{2}\right]$, the maximum part can be rewritten as Equation (5):

$$
\left(w_{c}^{T} x\right)^{2}=\max _{j}\left[\left(w_{j}^{T} x\right)^{2}\right]=\lim _{r \rightarrow \infty}\left[\sum_{j}\left(w_{j}^{T} x\right)^{2 r}\right]^{\frac{1}{r}}
$$

Let Equation (5) substitute in Eqn. (4) and let $B=\sum_{j}\left(w_{j}^{T} x\right)^{2 r}$, the cost function of WTA-ICA model is obtained

$$
J(W)=\int \lim _{r \rightarrow \infty}\left[\sum_{j}\left(w_{j}^{T} x\right)^{2 r}\right]^{\frac{1}{r}} p(x) d x=\int \lim _{r \rightarrow \infty} B^{\frac{1}{r}} p(x) d x
$$

\section{B. Updating Weights using WTA rule}

Using the classical gradient descent algorithm to solve the maximum of Eqn. (6), we can deduce the learning rule of the $j$ th column vector $w_{j}$ shown in Eqn. (7)

$$
\frac{\partial \boldsymbol{J}}{\partial w_{j}}=\int \lim _{r \rightarrow \infty} \frac{\partial \boldsymbol{B}_{r}^{\frac{1}{r}}}{\partial w_{j}} p(x) d x
$$

here $\left(\partial B^{\frac{1}{r}} / \partial_{w_{j}}\right)$ is deduced as follows

$$
\frac{\partial B^{\frac{1}{r}}}{\partial w_{j}}=\frac{1}{r} B^{\frac{r-1}{r}} \cdot \frac{\partial B}{\partial w_{j}}=2 B_{r}^{\frac{1}{r}} \cdot \frac{\left(w_{j}^{T} x\right)^{2 r-1}}{B} x
$$

where Eqn.(9) is the updating rule for the weight matrix $W$. Note that $\lim _{r \rightarrow \infty}\left[w_{j}^{T} x^{(2 r-1)} / B\right]=\delta_{c j}\left(w_{j}^{T} x\right)^{-1}$, where parameter $\delta_{c j}$ is the Kronecker delta [8]. If $c=j$, then $\delta_{c j}=1$, otherwise, $\delta_{c j}=0$. Thus, the limitation of Eqn. (10) was obtained as:

$$
\lim _{r \rightarrow \infty} \frac{\partial B^{\frac{1}{r}}}{\partial w_{j}}=2 \delta_{c j}\left(w_{c}^{T} x\right) x
$$

Assumed $\left(w_{j}^{T} x\right) x$ term with $\left\|w_{j}\right\|=1$ to be an observation, the goal is to get the man of this observation, while $w_{j}$ is estimated incrementally. The sample mean values uses a batch method, which is called an amnesic mean is defined as

$$
\left\{\begin{array}{l}
\bar{x}^{(n)}=\alpha(n) \bar{x}^{(n-1)}+\beta(n) x_{n} \\
\alpha(n)=\frac{n-1-\mu(n)}{n} \\
\beta(n)=\frac{1+\mu(n)}{n}
\end{array}\right.
$$

where $\bar{x}^{(n)}$ is the mean at the $n t h$ iteration, $x_{n}$ is the $n t h$ sample and $\mu(n)$ is a non-negative small function that discounts old estimate and gives more weights to the new observation $x_{n}$ at time $n$. Based on Eqns. (10) and (11), the updating rule of weight vector $w_{j}$ can be derived as:

$$
w_{j}\left(n_{j}+1\right)=\alpha\left(n_{j}\right) w_{j}\left(n_{j}\right)+\beta\left(n_{j}\right) \frac{w_{j}\left(n_{j}\right)^{T} x_{t}}{\left\|w_{j}\left(n_{j}\right)\right\|} x_{t}
$$


where $w_{j}\left(n_{j}\right)$ is the component vector $w_{j}$ after the $n_{j}$-th updating, $x_{t}$ is the current whitened data input.

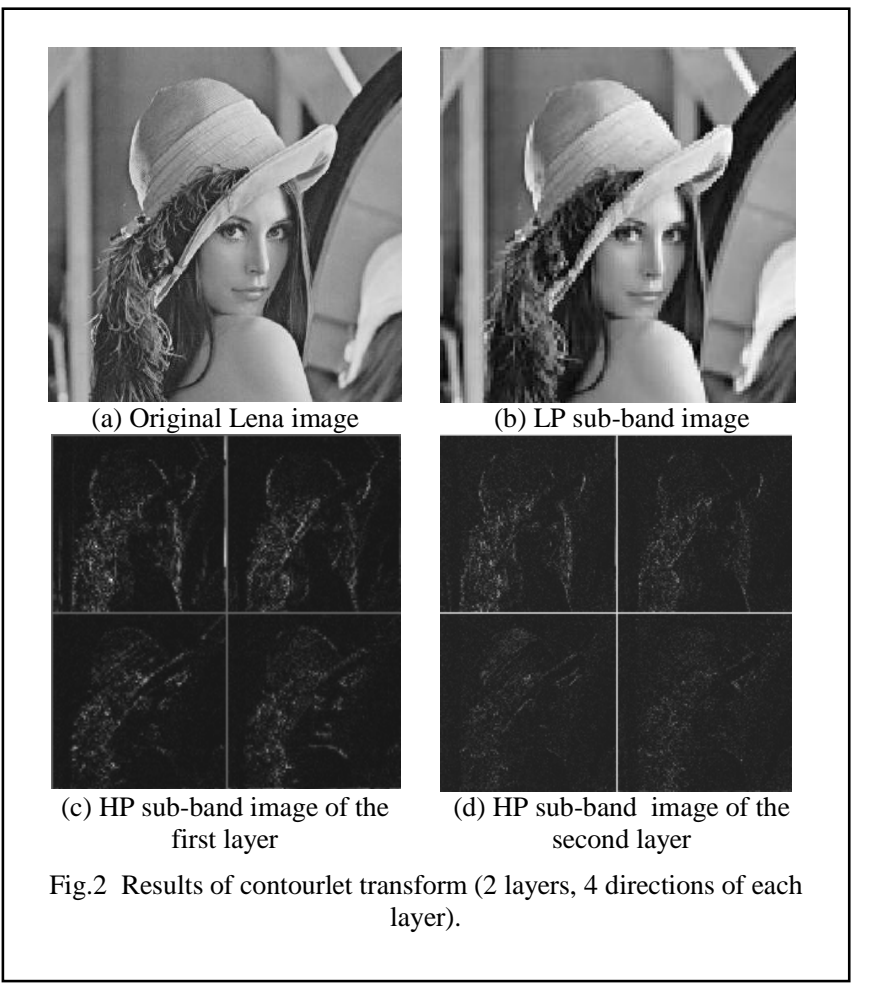

\section{EXPERIMENTAL RESULTS AND ANALYSIS}

\section{A. Sub-band Images Obtatined by Contourlet Transform}

Test images used in this paper were freely downloaded from http://sipi.usc.edu/database. Five images with $512 \times 512$ size were selected. And each image was first decomposed by contourlet transform. The number of decomposition layers and each layer's direction were supposed as 2 and 4 . For example, for Lena image, the decomposition results were shown in Fig. 2. Thus, for five original images, after contourlet transform, 5 LP sub-band images with $128 \times 128$ size were obtained and denoted by $X_{\text {Low }}$. At the same time, $20 \mathrm{HP}$ sub-band images of Layer 1 with $256 \times 256$ size and those of Layer 2 with $512 \times 512$ size were also obtained and denoted respectively by $X_{1 h i g h}$ and $X_{2 h i g h}$.

\section{B. Features Learning by WTA-ICA Model}

This sub-section discusses mainly feature learning of LP and HP sub-band images by using WTA-ICA model. To get input image data for WTA-ICA model, for each LP subband image, it was sampled randomly 10000 times with $8 \times 8$ patches, and for each HP sub-band images, it was sampled randomly 2500 times with the same image patch size. And then, each image patch was converted into a column to save. Thus, the size of $X_{\text {Low }}, X_{1 h i g h}$ and $X_{2 h i g h}$ was all $64 \times 50,000$. Using WTA-ICA model to train each data set, the corresponding LP feature bases, denoted by $A_{L P}$, and each layer's HP feature bases learned, denoted by $A_{H P 1}$ and $A_{H P 2}$ respectively, were shown in Fig.3 (b) to Fig.3 (d). As comparison, for the image patch set of five original clear test images used, the feature bases obtained by WTA-ICA were also shown in Fig.3 (a). From Fig.3, it can see that features of HP sub-band images have been extracted clearly.

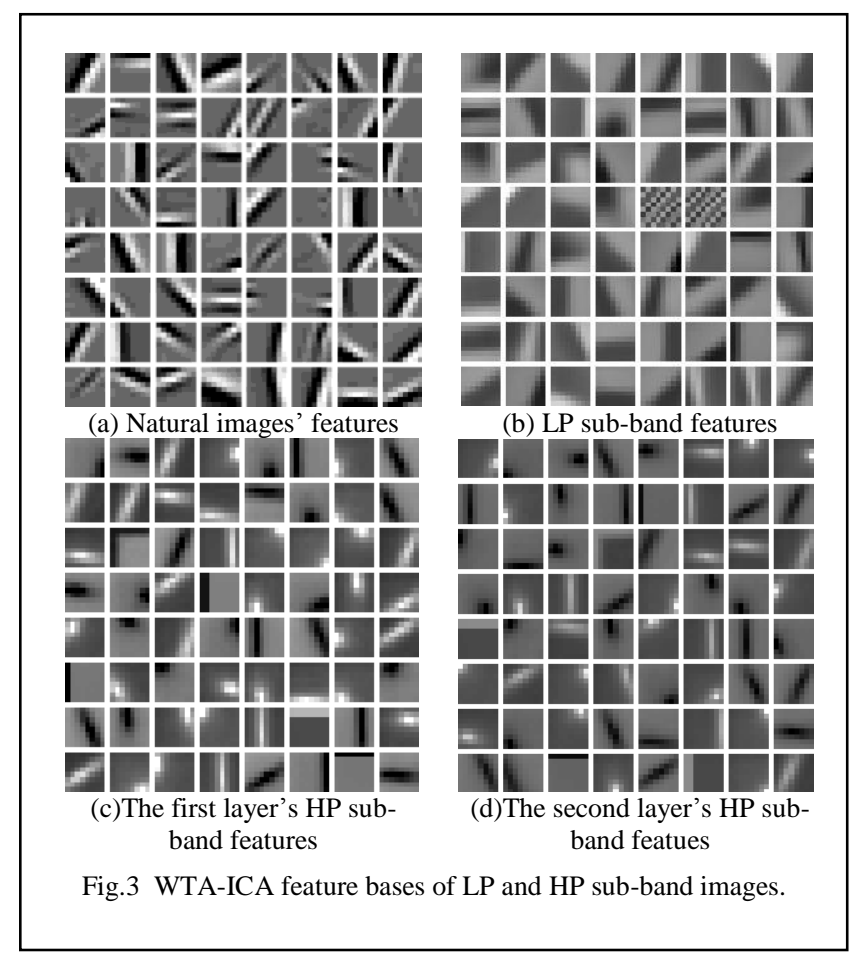

C. Image Reconstruction Based on Fusion Features

For each layer's features of HP sub-band images extracted by WTA-ICA model, the weighted average feature fusion technique was first utilized here, and the fusion features between the first layer's and the second layer's HP sub-band image features was denoted by $A_{H P}$. Further, for LP sub-band image features $A_{L P}$ and the fusion HP sub-band features $A_{H P}$, the fusion feature method based on kernel canonical correlation analysis (KCCA) [9] was discussed again. Thus, the total fusion features $A_{\text {total }}$ of test images were obtained. In the image reconstruction task, the test image was selected as the classical Lena image with $512 \times 512$ pixels. Assume that the number of image patches with $8 \times 8$ pixels sampled from Lena image was 1000,5000 , 10000 and 50000, respectively. Corresponding to different image patches, the image reconstruction results obtained by our algorithm were shown in Fig. 4. At the same time, the reconstruction ones of WTA-ICA model were also given in Fig. 5. Distinctly, from Fig.4 and Fig.5, it can be seen that the larger the number of image patches is, the clearer the reconstructed image is. Further, to testify the reconstructed effect of our method, the SNR criterion was used to measure 
the reconstructed effect obtained by WTA-ICA and our method. The SNR values were listed in Table 1.

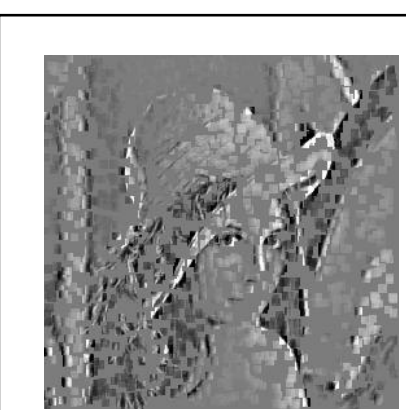

(a) 1000

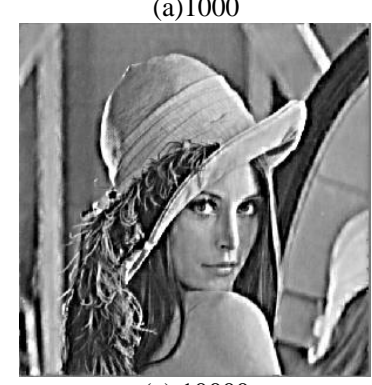

(c) 10000

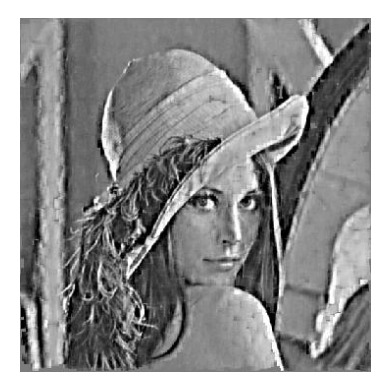

(b) 5000

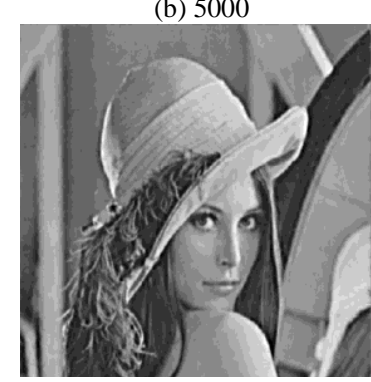

(d) $50000 \mathrm{r}$
Fig.4 Reconstructed results of our method with different image patches.

Table 1. Values of SNR obtained by different algorithms corresponding to different image patches.

\begin{tabular}{ccccc}
\hline \multirow{2}{*}{ Algorithms } & \multicolumn{4}{c}{ Image patches } \\
\cline { 2 - 5 } WTA-ICA & 1000 & 5000 & 10000 & 50000 \\
Our & 10.51 & 13.81 & 14.36 & 20.53 \\
method & 11.14 & 14.45 & 15.62 & 23.47 \\
\hline
\end{tabular}

\section{COPYRIGHT FORMS AND REPRINT ORDERS}

In this paper, an image reconstruction method based on feature fusion technique is developed by using WAT-ICA model in contourlet transform domain. For each layer's high frequency features, the weighted average feature fusion rule is used, further, for the fused high frequency features and the low frequency features, the KCCA fusion method is used and the total fused features can be obtained. Used the total fused features here, the image reconstruction task can be done. Experimental results testify that our method proposed is indeed efficient in reconstructing an image in application.

\section{ACKNOWLEDGMENT}

This work was supported by the National Natural Science Foundation of China (No. 61373098), the Innovative
Research Team of Suzhou Vocational University (No. 3100125).

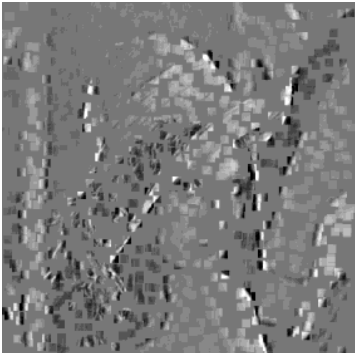

(a) 1000

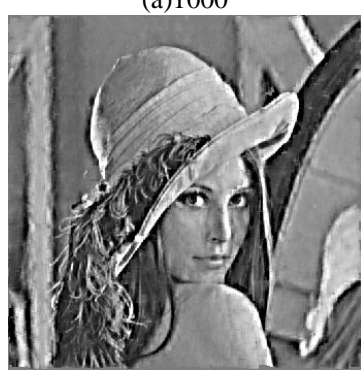

(c) 10000

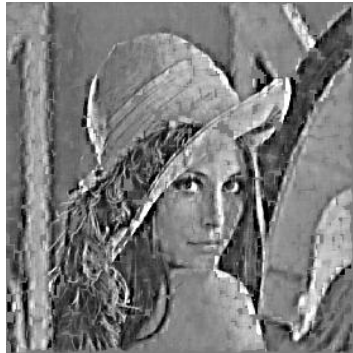

(b) 5000

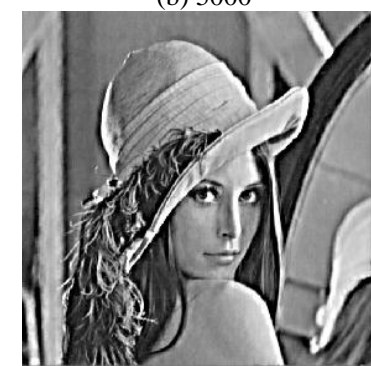

(d) $50000 \mathrm{r}$
Fig.5 Reconstructed results of WTA-ICA model with different image patches.

\section{References}

[1] D. L. Donoho, "Denoising by soft thresholding", IEEE Trans. On Information Theory, vol. 41, no.3, pp. 613-627, March 1995.

[2] B. K. Shreyamsha Kumar, "Image denosiign based on non-local means filter and its method noise thresholding", Signal, Image and Video Processing, vol.7, no.6, pp.1211-1227, Nov. 2013.

[3] D. L. Donoho and I. M. Johnstone, "Ideal spatial adaptation by wavelet shrinkage", Biometrika, vol. 81, no.3, pp. 425-455, March 1994.

[4] Michael Elad and Michal Aharon, "Image denoising via sparse and redundant representation over learned dictionaries", IEEE Trans. On Image Processing, vol. 15, no.12, pp. 3736-3745, December 2006.

[5] M. N. Do and M. Vetterli, "The contourlet transform: an efficient directional multiresolution image representation", IEEE Trans. On Image Processing, vol.14, no. 12, pp. 2091-2106, Dec. 2006.

[6] E. J. Candès, L. Demanet, D. L. Donoho and L. Ying, "Fast discrete curvelet transforms", Tech. Rep., Applied and Computational Mathematics, Califormia Institure of Technology, 2005.

[7] Li Shang, Pin-gang Su and Tao Liu, "Denoising MMW image using the combination method of contourlet and KSC shrinkage", Neurocomputing, vol. 83, pp. 229-233, Apr. 2012.

[8] N. Zhang and J. Weng, "Sparse representation from a winner-take-all neural network", Proc. Of IEEE International Joint Conference on Neural Networks (IJCNN 04), IEEE Press, New Work, USA, pp. 2209-2214, July 2004.

[9] Xiaona Xu and Zhichun Mu, "Feature fusion method based on KCCA for ear and profile face based multimodal recognition", Proc. of IEEE International Conference on Automation and Logistics IEEE Press, New Work, USA, pp. 620-623, Aug. 2007. 